\title{
Análisis de las características fisicoquímicas de dos tipos de suelos ubicados en la vía Becerril, La Jagua de Ibirico en el departamento del Cesar
}

Daniel Andrés Cotes García' Luis Díaz Muegue²

\section{Resumen}

El suelo es un cuerpo natural diferenciado en horizontes de mineral y constituyentes orgánicos, usualmente no consolidados, de profundidad variable, los cuales difieren del material parental de abajo en morfología, propiedades físicas y características biológicas. El objetivo general de este estudio es analizar las características fisicoquímicas de dos tipos de suelos ubicados en la vía Becerril, La Jagua de Ibirico en el departamento del Cesar. Está basado en una visita de campo realizada a estas zonas. Con los resultados obtenidos se concluye que los suelos de la zona centro del departamento del Cesar son muy productivos, los cuales se pueden utilizar para desarrollar diferentes actividades agrícolas que permitan que los habitantes de la región puedan encontrar una alternativa de generación de ingresos diferente a la actividad minera.

Palabras clave: características fisicoquímicas, descripción de suelos, suelo.

\footnotetext{
'Ingeniero de minas, estudiante de Maestría en Ciencias Ambientales SUE Caribe, docente de la Fundación Universitaria del Área Andina, sede Valledupar. Correo: dacotes(areandina.edu.co

${ }^{2}$ Ingeniero de Minas, docente de la Maestría en Ciencias Ambientales SUE Caribe, gestor de proyectos de investigación de la Fundación Universitaria del Área Andina, sede Valledupar. Correo: Idiaz164Careandina.edu.co
} 


\section{Introducción}

Teniendo en cuenta lo planteado por Jofpe (1936), citado por Jenny (1994), "el suelo es un cuerpo natural, diferenciado en horizontes de mineral y constituyentes orgánicos, usualmente no consolidados, de profundidad variable, los cuales difieren del material parental de abajo en morfología, propiedades físicas y características biológicas".

El departamento del Cesar se encuentra influenciado por dos sistemas montañosos, la Sierra Nevada de Santa Marta y la Serranía del Perijá, en este estudio se tomará como referencia la Serranía del Perijá. Según un estudio realizado por Rangel, Carvajal y Arellano,

la caracterización físico-química de los suelos a lo largo del gradiente altitudinal de este sistema, muestra que en la alta montaña hay Entisoles (Lithic Troporthent y Typic Ustorthent) e Inceptisoles (Aquic Humitropept, Entic Dystropept, Typic Humitropept y Vertic Eutropept) cuyos parámetros químicos denotan condiciones de bajas en el contenido de elementos y condiciones o propiedades. En la media montaña dominan los Inceptisoles (Andic Humitropept, Typic Dystrandept, Entic Dystro- pepts) y Entisoles (Typic Troporthent), con textura Franco-Arenosa, con contenidos de Calcio, Potasio, Fósforo y Carbono son bajos. En la baja montaña predominan los Inceptisoles (Typic Dystropepts y Typic Humitropept), ocasionalmente, se encuentran Entisoles (Tropofluventy Typic Troporthents) con predominio de las texturas Franco-Arenosa (FA) y Franca (F). Y en la región tropical predominan Entisoles (Typic Ustorthens y Tropofl uvents) e Inceptisoles (Typic Eutropepts) que tienen texturas Franca y Franca-Arenosa.

La geología de las dos zonas escogidas para este estudio corresponde a la formación Rionegro, esta formación, según Hernández (2003, citado por Universidad Nacional y ANH, 2009),

es detrítica, de composición especialmente arcósica. En el cerro Arenas Blancas, al noroccidente de Poponte, está conformada por capas de arenisca de grano grueso, arenisca conglomerática y conglomerado; las capas son delgadas y en algunas se observa estratificación cruzada. La formación Río Negro suprayace discordantemente capas de la formación La Quinta e infrayace de forma transicional al Grupo Cogollo. 
Por sus características litológicas, Hernández (2003, citado por Universidad Nacional y ANH, 2009) "ha postulado un ambiente de depósito que corresponde a abanicos aluviales, los cuales drenaban desde los altos del basamento (Formación La Quinta y rocas paleozoicas) ubicadas al oeste, este y norte de la actual".

La serranía del Perijá está compuesta por rocas metamórficas del Paleozoico sin diferenciar pizarras, filitas, esquistos verdes y negros, cuarcita y mármol; también se presentan rocas sedimentarias del Devónico medio al Pérmico, principalmente conglomerados, areniscas rojisas, shales rojo y verde, caliza fosilífera y arenisca cuarzosa. También se encuentra evaporita, roca fosfórica y carbón (IGAC, 1997).

En lo que a vegetación se refiere las zonas de estudio están comprendidas en la vía que comunica a los municipios de Becerril y La Jagua de Ibirico, en la gran mayoría de la extensión territorial del municipio de Becerril, se encuentra la formación vegetal denominada bosque seco tropical, con alturas hasta 1000 metros sobre el nivel del mar, temperatura media de $28^{\circ} \mathrm{C}$, lo cual hace una región apta para el desarrollo ganadero y para la agricultura con riego suplementario.
La vegetación ha desaparecido en gran parte para dar paso a los pastizales, sin embargo, se observan vestigios de esta vegetación en algunos sectores de la planicie y aledañas a los ríos. (Alcaldía Municipal de Becerril, 2016)

"Por otro lado, los bosques de La Jagua de Ibirico se clasifican en cuatro tipos específicos: i) bosque seco tropical, ii) bosque húmedo tropical, iii) bosque muy húmedo premontano bajo y iv) bosque húmedo premontano" (Red Ormet, PNUD).

El clima en el municipio de La Jagua de Ibirico es muy similar al del municipio de Becerril, es cálido con temperaturas que van desde los $24^{\circ} \mathrm{C}$ hasta $\operatorname{los} 40^{\circ} \mathrm{C}$ en las partes bajas, con una temperatura media de $28^{\circ} \mathrm{C}$. El régimen de lluvias es bimodal. (Red Ormet, PNUD)

Este estudio es importante porque permite tener una descripción de suelos detallada de dos puntos ubicados en la zona minera del centro del departamento del Cesar, lo que permitirá conocer los usos de suelos más óptimos y que brinden alternativas distintas a las de la minería. Para ello se planteó como objetivo general analizar las características fisicoquímicas de dos tipos de suelos ubicados en la vía Becerril, La Jagua de 
Ibirico en el departamento del Cesar, basado en una visita de campo realizada a estas zonas.

\section{Materiales y métodos}

\section{Localización geográfica}

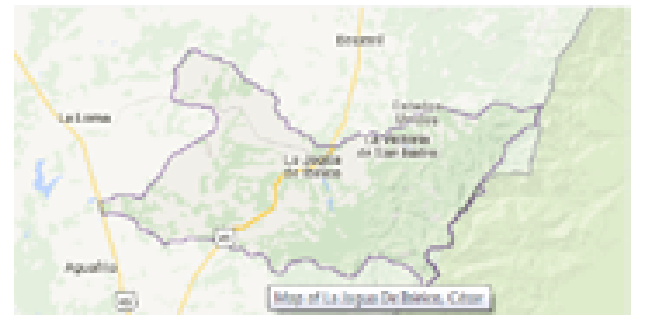

Figura 1. Localización de la zona de estudio

Fuente:https://plus.google. com/112363144376623532029

La zona de estudio corresponde a dos estaciones ubicadas en la vía Becerril, La Jagua de Ibirico, la primera se ubica al norte del municipio de, en la zona Boquerón, El Hatillo, la cual se encuentra ubicada al lado de la línea férrea y se está influenciada por la actividad minera de las empresas carboníferas.

El municipio está formado por dos tipos de paisajes como son la Serranía del Perijá que corresponde a la parte alta y montañosa hacia el oriente con elevaciones que superan los 2.900 metros sobre el nivel del mar y es la línea fronteriza con Venezuela, el otro paisaje es la planicie del pie de monte en el cual se encuentran localizados los yacimientos carboníferos". (Alcaldía La Jagua de Ibirico, 2012)

"El clima en el municipio es cálido con temperaturas que van desde los $24^{\circ} \mathrm{C}$ hasta $\operatorname{los} 40^{\circ} \mathrm{C}$ en las partes bajas, con una temperatura media de $28^{\circ} \mathrm{C}$. El régimen de lluvias es bimodal". (Red Ormet, PNUD)

La segunda estación se encuentra en el corregimiento Estados Unidos, el cual pertenece al municipio de Becerril, el clima del municipio es cálido tropical con humedad variable de cálido a seco, influenciado por los vientos alisios que se presentan con regularidad en los meses de diciembre a marzo dando origen a los periodos secos y de abril a noviembre periodo de lluvias que se hacen más intensos en septiembre a noviembre, y 22 precipitaciones de $961 \mathrm{~mm}$. La temperatura promedio es de $28^{\circ}$ en las zonas planas y de $10^{\circ} \mathrm{C}$ en la serranía del Perijá, con una humedad relativa del $76 \%$, precipitación $961 \mathrm{~mm}$. (Secretaría Departamental de Salud del Cesar, 2010) 


\section{Metodología de caracterización físicoquímica realizada a las muestras de suelo}

Para caracterizar el $\mathrm{pH}$ y la conductividad eléctrica fueron medidos en proporción suelo-agua 1:1 y 1:5, respectivamente. Para medir el $\mathrm{pH}$ en campo se utilizó un pHmetro portátil en una suspensión de suelo, la solución se agitó y se dejó reposar por quince minutos, después se leyó el valor del pH. (FAO, 2009) Para el análisis de los valores de $\mathrm{pH}$ se tuvo en cuenta la tabla 1 :
El carbono orgánico del suelo (COS) fue determinado por el método de oxidación húmeda con dicromato potásico conocido como método Walkley-Black. El contenido de materia orgánica de los horizontes minerales se estimó mediante el color del suelo seco y/o húmedo en la tabla Munsell, tomando en cuenta su clase textural (tabla 2). Esta estimación se basa en la suposición de que el color del suelo (valor) es debido a la mezcla de sustancias orgánicas de color oscuro y minerales de color claro. (FAO, 2009)

Tabla 1. Clasificación de los valores de pH

\section{Clasificación de los valores de $\mathrm{pH}$}

\begin{tabular}{|c|c|c|}
\hline \multirow[t]{6}{*}{$\mathrm{pH}_{\mathrm{CrCl}_{2}}$ de } & $<b .1,51>13 \% \mathrm{MU}$ & $\begin{array}{l}\text { es una indicacion para un } \\
\text { calificador Districo (= saturacion } \\
\text { de base) }\end{array}$ \\
\hline & $<4.6$, si $4-15 \% \mathrm{MO}$ & \\
\hline & $<4.2$, si $<4 \% \mathrm{MO}$ & $\begin{array}{l}\text { ( }<50 \%) \text {, de otro modo } \rightarrow \\
\text { calificador Etrico }\end{array}$ \\
\hline & $<3.6$, si $>15 \% \mathrm{MO}$ & $\begin{array}{l}\text { es una indicación para una } \\
\text { saturación de bases menor de } \\
10 \%\end{array}$ \\
\hline & $<3.4$, si $4-15 \% \mathrm{MO}$ & \\
\hline & $<3.2$, si < 4\% MO & $\begin{array}{l}\text { y para una alta saturación de Al } \\
\rightarrow \text { calificador Hiperálico }\end{array}$ \\
\hline
\end{tabular}

Fuente: Adaptado de Schlichting, Blume y Stahr (1995). 
Tabla 2. Estimación del contenido de materia orgánica basada en el color del suelo de Munsell

\begin{tabular}{|c|c|c|c|c|c|c|c|}
\hline \multirow[t]{3}{*}{ Color } & \multirow{3}{*}{$\begin{array}{l}\text { Vabor } \\
\text { Munsell }\end{array}$} & \multicolumn{3}{|c|}{ Sueto humedo } & \multicolumn{3}{|c|}{ Suelo seco } \\
\hline & & A & $A$, IA, I & 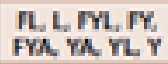 & A & AI, TA, I & 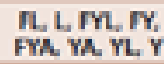 \\
\hline & & \multicolumn{6}{|c|}{ (*) } \\
\hline Gris claro & 7 & & & & $<0,3$ & $<0.5$ & $<0.6$ \\
\hline Gris claro & 6.5 & & & & $0.3-0.6$ & $0.5-0.8$ & $0.6-1.2$ \\
\hline Grit & 6 & & & & $0,6-1$ & $0,8-1,2$ & $1,2-2$ \\
\hline Grik & 5,5 & & & $<0,3$ & $1=1,5$ & $1,2-2$ & $2-3$ \\
\hline Gris & 5 & $<0,3$ & $<0,4$ & $0,3-0,6$ & $1,5-2$ & $2-4$ & 3.4 \\
\hline Grib oscuro & 4,5 & $0,3-0,6$ & $0,4-0,6$ & $0,6-0,9$ & $2-3$ & $4-6$ & $4-6$ \\
\hline Grit oscuro & 4 & $0,6-0,9$ & $0,6-1$ & $0,9-1,5$ & 3-5 & $6-9$ & 6-9 \\
\hline Grit negro & 3,5 & $0,9-1,5$ & $1-2$ & $1,5-3$ & $5-8$ & 9-15 & $9-15$ \\
\hline Gris negro & 3 & $1.5-3$ & $2-4$ & $3-5$ & 8-12 & $>15$ & $>15$ \\
\hline Negre & 2.5 & $3-6$ & $>4$ & $>5$ & $>12$ & & \\
\hline Negre & 2 & $>6$ & & & & & \\
\hline
\end{tabular}

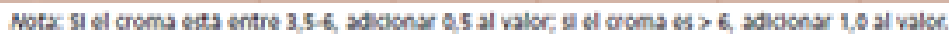

Puente: Adaptaso de schecteng. Blame y stahr (1965).

"El fósforo se cuantificó por el método Olsen. La extracción del fósforo se hizo con solución de bicarbonato de sodio $0,5 \mathrm{~N}$ a $\mathrm{Ph} 8,5$ " (Andrades, Moliner y Masaguer, 2015, p. ). En un tubo con tapa se colocaron $2,5 \mathrm{~g}$ de suelo $\mathrm{y}$ se agregó $30 \mathrm{ml}$ de la solución, luego se agitó por treinta minutos y se filtraron las muestras en tubos de ensayo, posteriormente se tomó $5 \mathrm{ml}$ de la solución filtrada con una pipeta automática y se añadió $1 \mathrm{ml}$ de $\mathrm{H} 2 \mathrm{SO} 4$ (2,5M), además, se agregaron $10 \mathrm{ml}$ de la solución de trabajo para el desarrollo de color con una repipeta y se esperó durante quince minutos, finalmente se calibró el espec- trofotómetro, se leyeron las muestras y se calculó la concentración de fósforo (Andrades, Moliner y Masaguer, 2015).

La capacidad de intercambio catiónico es una medida de la cantidad de cationes intercambiables que están neutralizando la carga negativa del suelo. Para el cálculo de esto se pesan $10 \mathrm{~g}$ de suelo, al cual se le añade $50 \mathrm{ml}$ de $\mathrm{KCl} 1 \mathrm{M}$ y se agita durante cinco minutos, luego se filtra por gravedad en balones de 100 $\mathrm{ml}$, lavando el suelo con cinco porciones de $10 \mathrm{ml}$ de $\mathrm{KCl}$, posteriormente se completa a volumen con $\mathrm{KCl}$ y se agita para obtener una mezcla homogénea; finalmente, este filtrado constituye el ex- 
tracto a partir del cual se determinan los cationes intercambiables $\mathrm{Ca}$ y $\mathrm{Mg}$ por absorción atómica (Andrades, Moliner y Masaguer, 2015).

La densidad real y aparente se calculó empleando el método propuesto por la NTC 5167, el método se basa en la cuantificación de la masa de producto que se deposita libremente por unidad volumen, en un recipiente de volumen conocido, primero se pesa la probeta vacía, limpia y seca, luego se deja caer libremente una cantidad de material suficiente para obtener una lectura cercana a $30 \mathrm{~cm} 3$, posteriormente se registra el volumen ocupado por el material y se determina el peso de la probeta con el material, finalmente se realizan tres determinaciones sobre muestras diferentes. (Icontec, 2004)

La textura se determinó por el método Boyoucus, este método requiere del acetato de amonio $1 \mathrm{~N} \mathrm{pH} \mathrm{7,0,} \mathrm{se}$ basa en el reemplazo de las posiciones de intercambio por el ión amonio y su posterior desplazamiento con una solución de cloruro de sodio, produciendo cloruro de amonio el cual en presencia de formaldehído forma estequiométricamente un complejo nitrogenado y ácido clorhídrico el cual es evaluado con hidróxido de sodio. (Andrades, Moliner y Masaguer, 2015)

El procedimiento utilizado consistió en pesar $40 \mathrm{~g}$ de tierra fina al aire en un vaso de precipitados de $250 \mathrm{ml}$, se añadieron $100 \mathrm{ml}$ de la solución dispersante y se dejó diez minutos, se añadió agua hasta completar los $250 \mathrm{ml}$ y se batió durante cinco minutos. Luego, se transfirió el contenido del vaso de precipitado a una probeta de un litro, poniendo en la boca de la probeta un embudo de boca grande y encima un tamiz de malla de $0,2 \mathrm{~mm}$ para separar la arena mayor de este tamaño.

El color del suelo por notación Munsell, según lo establecido en el manual de la FAO, el cual muestra que el contenido de materia orgánica de los horizontes minerales se puede estimar mediante el color del suelo seco y/o húmedo en la tabla Munsell, tomando en cuenta su clase textural. Esta estimación se basa en la suposición de que el color del suelo (valor) es debido a la mezcla de sustancias orgánicas de color oscuro y minerales de color claro. (FAO, 2009) 
La presencia de carbonatos se calculó tomando como referencia lo planteado por el manual de la FAO, esta se estableció a través de la adición al suelo de gotas de $\mathrm{HCl}$ (Ácido Clorhídrico) al 10 por ciento, donde el grado de efervescencia del gas de dióxido de carbono es indicativo de la cantidad de Carbonato de Calcio presente en el suelo. (FAO, 2009)

El tipo de Carbonato encontrado se definió con base en la siguiente tabla:

Tabla 3. Clasificación de la reacción del carbonato en la matriz del suelo

\begin{tabular}{|c|c|c|c|}
\hline & $\%$ & & \\
\hline N & 0 & No calcáreo & $\begin{array}{l}\text { No detectable visiblemente } \\
\text { ni efer vescencia dudible }\end{array}$ \\
\hline SL & $\approx 0-2$ & Ligeramente calcareo & $\begin{array}{l}\text { Se escucha la etervescencia } \\
\text { peru nu ts visible }\end{array}$ \\
\hline MO & $\approx 2-10$ & $\begin{array}{l}\text { Moderadamente } \\
\text { calúreu }\end{array}$ & Efervescencla vislble \\
\hline ST & $\approx 10-25$ & Fuertemente calcareo & $\begin{array}{l}\text { Efervescencla fuertemente } \\
\text { visible. Las burbujas } \\
\text { forman poca cspuma }\end{array}$ \\
\hline [X & $\mu>25$ & $\begin{array}{l}\text { Lxtremadamente } \\
\text { calcároo }\end{array}$ & $\begin{array}{l}\text { Reacción extremadamente } \\
\text { fuorto. So forma la ospuma } \\
\text { espesa rápidamente }\end{array}$ \\
\hline
\end{tabular}

\section{Resultados}

\section{Estación 1. Zona Boquerón, El Hatillo CNR}

En esta estación se evidenció la presencia de suelos originados de terrazas aluviales, se encontró cuarzo y gran contenido de óxidos de hierro, lo que puede indicar que este corresponde a un plintosol. En la figura 1 se presenta la evidencia de una plintita, la cual es una roca pesada debido a la gran cantidad de óxido de hierro. 


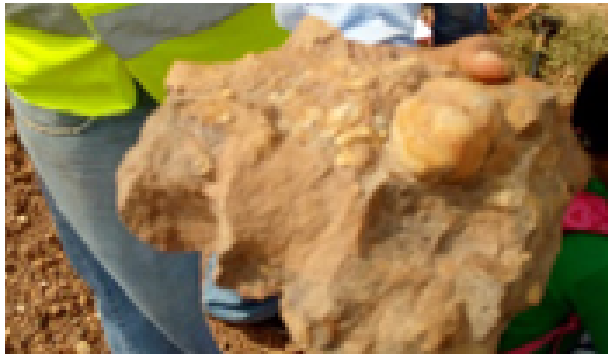

Figura 1. Plintita

La vegetación predominante en la zona es peralejo, pasto carimagua.

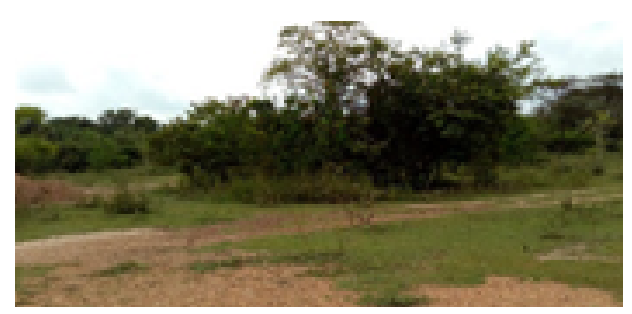

Figura 2. A. Vegetación de la estación Boquerón, El Hatillo

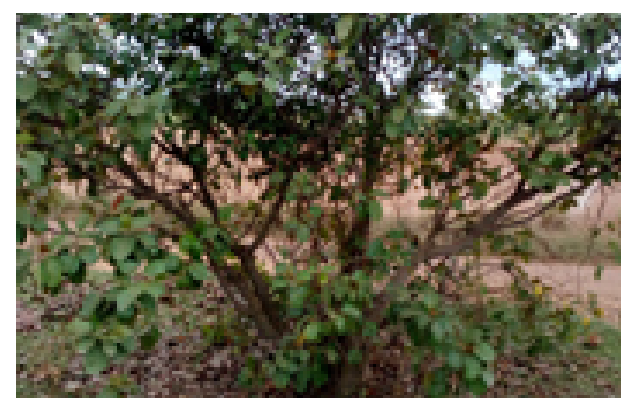

Figura 2.B. Vegetación de la estación Boquerón, El Hatillo

Se observó la presencia de tres horizontes de suelo, en el cual el horizonte B corresponde a arcilla.
El agua baja hasta el nivel de la arcilla, por ausencia de oxígeno se presentan los colores grises. La textura es completamente arcillosa debido a la humedad.

En el horizonte A hay presencia de suelos, mayor cantidad de rocas o elementos pétreos. Es muy pedregoso porque tiene bastante cantidad de elementos gruesos.

Moteado del suelo. Indica cambios en el suelo por presencia de humedad o secado.

Se observó la presencia de suelos rojos, suelos con alto contenido de hierro, tal como aparece en la figura 3.

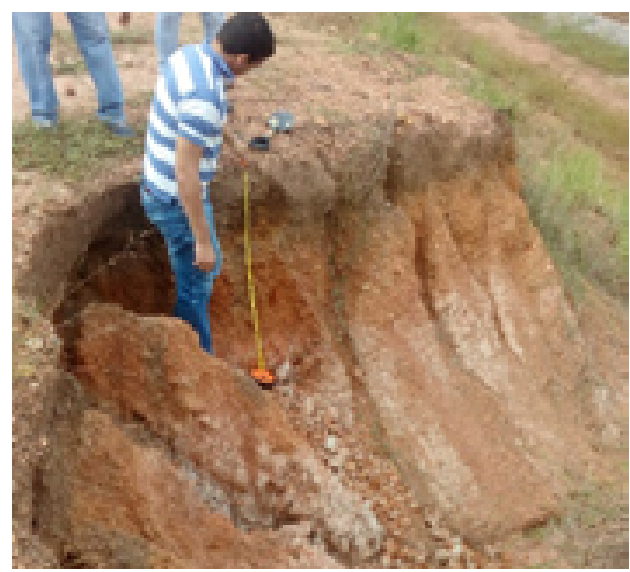

Figura 3. Suelos de la estación Boquerón, El Hatillo

Horizonte A: $40 \mathrm{~cm}$, material granular melanizado, textura con presencia de bioturbación de raíces 
Horizonte B: $50 \mathrm{~cm}$, material granular, textura con presencia de bioturbación de raíces hasta los primeros $25 \mathrm{~cm}$ de iniciado el horizonte

Horizonte C: a mayor profundidad de $1 \mathrm{~m}$ con moteados de hierro y textura arcillosa

Pendiente: $10^{\circ}$

\begin{tabular}{|r|c|c|}
\hline \multirow{2}{*}{$1,30 \mathrm{~m}$} & A & $0,40 \mathrm{~m}$ \\
\hline & B & $0,50 \mathrm{~m}$ \\
\hline & C & $0,40 \mathrm{~m}$ \\
\hline
\end{tabular}

Figura 4. Perfil de suelo, estación Boquerón, El Hatillo

Se tomó el color en seco y en húmedo con la tabla Munsell.
Horizonte A. 2,5 YR 4//4

Horizonte B. 2,5 YR 4//6

Horizonte C. 2,5 YR 5//8

Coordenadas

N 9०37'50,3"

W $73^{\circ} 32^{\prime} 51,7^{\prime}$

Altura $59 \mathrm{~m}$

TA. $30,3{ }^{\circ} \mathrm{C}$

Se cataloga como horizonte B porque tiene presencia de raíces

TB. $28,7^{\circ} \mathrm{C}$

TC. $28,8^{\circ} \mathrm{C}$

Hay lixiviación de hierro

\section{En seco}

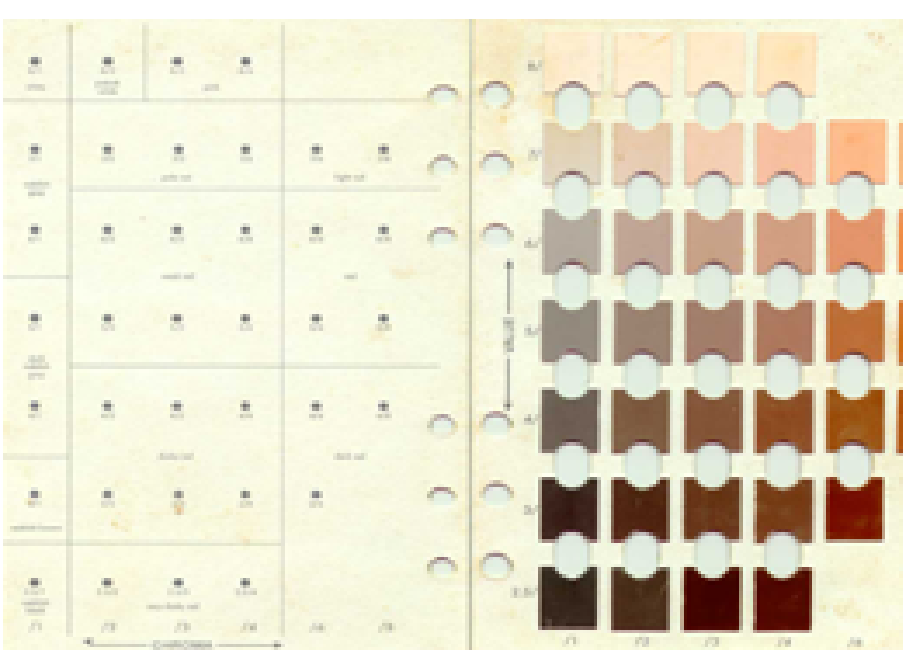




\section{En húmedo}

Horizonte A. 2,5 YR 3//1

Horizonte B. 2,5 YR 4//8

Horizonte C. 2,5 YR 5//8

T Ambiente. $30,8^{\circ} \mathrm{C}$

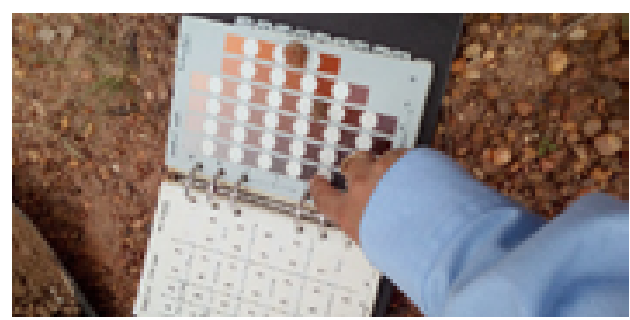

Figura 6. Identificación del color del suelo

$$
\begin{aligned}
& \text { pH A. } 5 \\
& \text { pH B. } 6 \\
& \text { pH C. } 5
\end{aligned}
$$

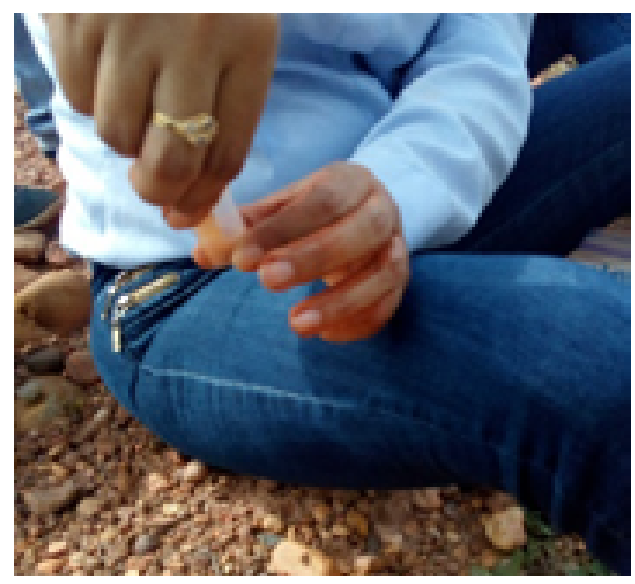

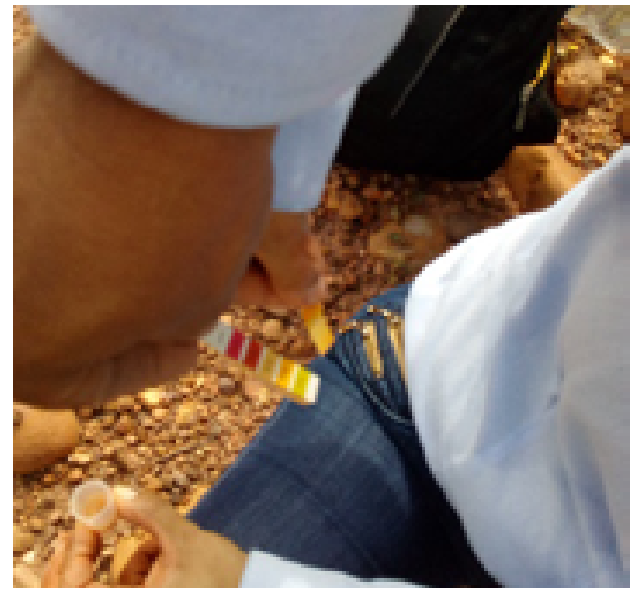

Figura 7. Determinación del pH del suelo

Textura A. Sin agua, arenoso

Textura B. Combinación de arcilla y granular, no se deja manejar porque tiene material granular

Textura C. Arcilla moldeable (con agua y sin agua)

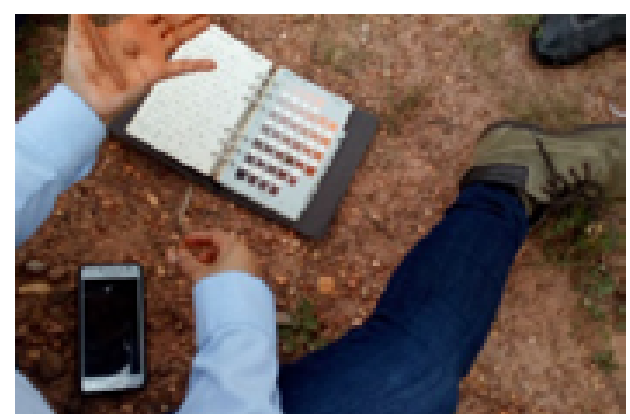

Figura 8. Determinación de la textura del suelo

Hay lixiviación de hierro en las arcillas 
Prueba de carbonatos. Se utilizó HCL (ácido clorhídrico) cinco gotas.

HA. Sin presencia de carbonatos

HB. Sin presencia de carbonatos

HC. Sin presencia de carbonatos

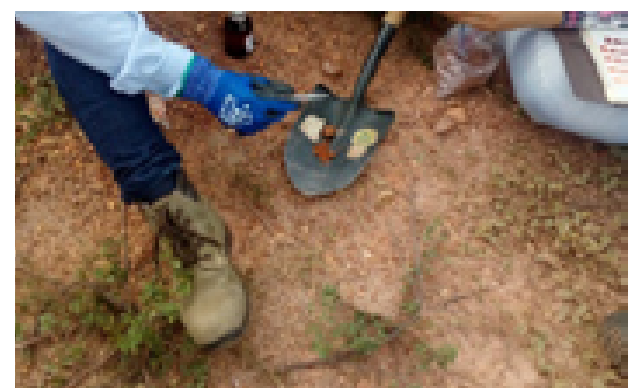

Figura 9. Determinación de la presencia de Carbonatos en el suelo

Peróxidos 5 gotas

HA. No hay efervescencia, bajo en materia orgánica

HB. Bajo en materia orgánica

HC. Bajo en materia orgánica

\section{Estación 2. Corregimiento de Estados Unidos}

Dentro de la vegetación encontrada se destaca la presencia de Moco de Hierro, hay mucha rocosidad, principalmente rocas grandes, arborización secundaria de mayor tamaño, boque seco tropical, tal como se muestra en la figura 10.

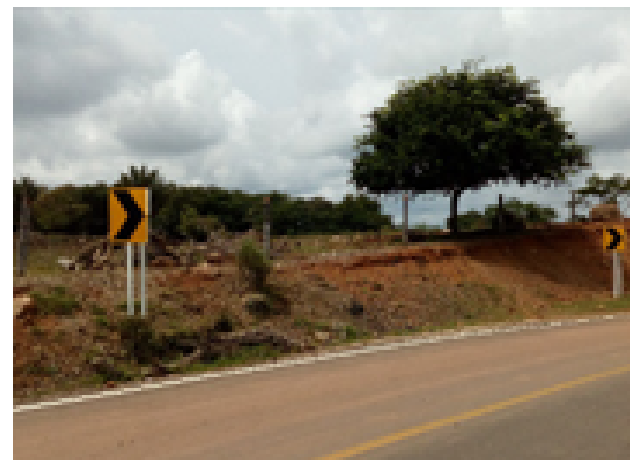

Figura 10. Vegetación del corregimiento Estados Unidos

\section{Coordenadas}

N 9 $38^{\prime} 56,7^{\prime \prime}$

$\mathrm{W} 73^{\circ} 16^{\prime} 34,3^{\prime \prime}$

Altura $141 \mathrm{~m}$

Se evidenció la presencia de bioturbación, debido a la presencia de lombrices, tal como se muestra en la figura 11; esto muestra que la salud del suelo es buena.

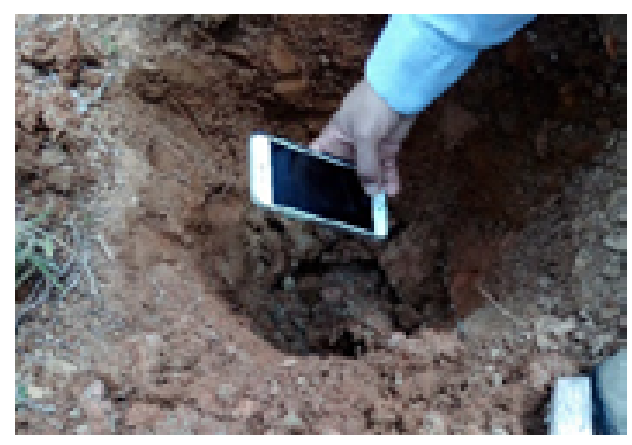

Figura 11. Presencia de lombrices en el suelo 
Temperatura ambiental, $36,2^{\circ} \mathrm{C}$

\begin{tabular}{|c|c|c|}
\hline \multirow{2}{*}{$0,60 \mathrm{~m}$} & A & $0,20 \mathrm{~m}$ \\
\cline { 2 - 3 } & B1 & $0,10 \mathrm{~m}$ \\
\cline { 2 - 3 } & B2 & $0,30 \mathrm{~m}$ \\
\hline
\end{tabular}

Figura 12. Perfil de suelo. Estación 2

Horizontes A y B

Horizonte $\mathrm{C}$ de piedras, porque hay caliches

A mayor profundidad el color es más gris. Significa reducción, ausencia de aire Hay moteados en el horizonte B

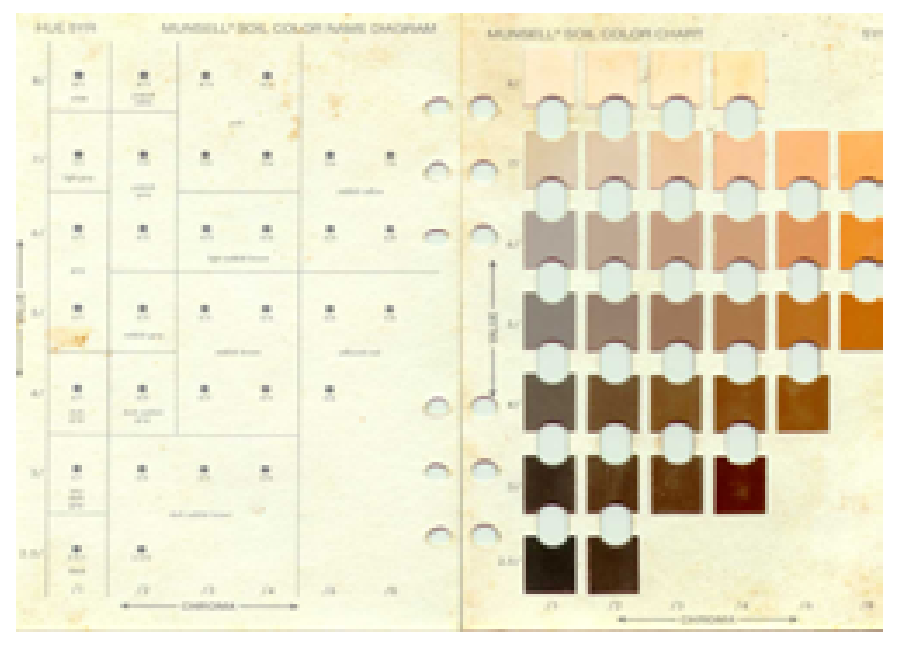

Figura 13. Tabla Munsell: Matiz 5YR

Seco

HA. 5YR 4/ /4

HB1. 5YR 5//4 
HB2. 5YR 6/ /4

\section{Húmedo}

HA. 5YR 4/ /3

HB1. 5YR 5//6

HB2. 5YR 6/ /6

La bolita grande corresponde al horizonte B1 y la bolita pequeña al horizonte B2, tal como se presenta en la figura 14 .

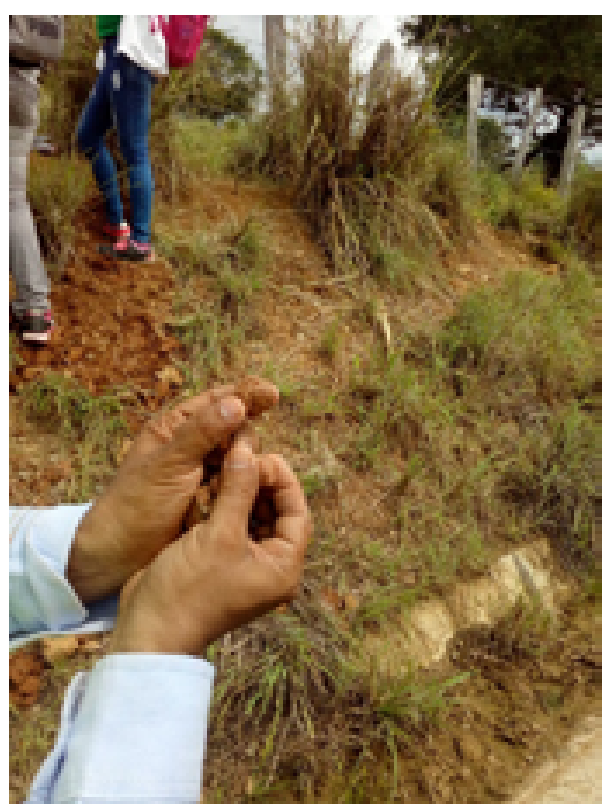

Figura 14. Determinación de la textura del suelo de la estación 2

TA. $31,5^{\circ} \mathrm{C}$
Pha.5

TB1. $31^{\circ} \mathrm{C}$

PhB1. 5

Presencia de hormigas. Salen del perfil B2

TB2. $31,6^{\circ} \mathrm{C}$

pH B2. 5

Textura A. Franco (en el laboratorio se especifica puntualmente la textura)

Textura B1. Arcillosa

Textura B2. Arcillosa

Carbonatos HCL

HA. No hay efervescencia, es básico

HB1. No hay efervescencia

Peróxidos 8 gotas

HA. Hay efervescencia, hay presencia de carbono orgánico

HB1. Hay mayor cantidad de carbono orgánico

HB2. Hay poco carbono orgánico

A continuación, se presentan los resultados obtenidos en laboratorio. 
Tabla 4. Características físico químicas

\begin{tabular}{|c|c|c|c|c|c|c|c|}
\hline \multirow{3}{*}{ Caracteristica } & \multirow{3}{*}{ Unidad } & \multicolumn{6}{|c|}{ Horizontes } \\
\hline & & \multicolumn{3}{|c|}{ Primer Punto } & \multicolumn{3}{|c|}{ Segundo Punto } \\
\hline & & A & B & C & $\mathbf{A}$ & B1 & B2 \\
\hline Color del suelo & Notación Munsell & & & & & & \\
\hline Tipo texura & & Arena & Fr-Arc-Are & Arcilla & Arc-Are & Arcilla & Arcilla \\
\hline Arena & $\%$ & 73,19 & 63,17 & 25,32 & 61,21 & 30,70 & 33,59 \\
\hline Arcilla & $\%$ & 14,24 & 28,89 & 61,17 & 35,49 & 56,77 & 61,30 \\
\hline Limo & $\%$ & 12,58 & 7,94 & 13,51 & 3,30 & 12,54 & 5,11 \\
\hline Densidad Aparente & $\mathrm{g} / \mathrm{cm} 3$ & 1,31 & 1,09 & 1,39 & 1,05 & 1,25 & 1,44 \\
\hline Densidad Real & $\mathrm{g} / \mathrm{cm} 3$ & 2,34 & 2,08 & 2,73 & 2,66 & 2,56 & 2,73 \\
\hline Porosidad & $\%$ & 43,86 & 47,61 & 49,04 & 60,59 & 51,09 & 47,38 \\
\hline Tamaño de poros & $\mathrm{mm}$ & 0,01 & 0,05 & 0,59 & 0,08 & 0,47 & 0,31 \\
\hline Humedad del suelo & $\%$ & 0,61 & 4,86 & 42,23 & 7,96 & 37,43 & 21,30 \\
\hline $\mathrm{pH}(1: 1)$ & Unidad de $\mathrm{pH}$ & 4,49 & 5,03 & 4,66 & 5,22 & 5,30 & 4,76 \\
\hline Conductividad Electrica & $\mathrm{dS} / \mathrm{m}$ & 0,06 & 0,05 & 0,05 & 0,06 & 0,05 & 0,12 \\
\hline $\mathrm{CIC}$ & $1 \mathrm{meq} / 100 \mathrm{~g}$ & 12,91 & 17,65 & 31,12 & 46,96 & 31,25 & 17,42 \\
\hline Carbono Organico & $\%$ & 3,76 & 2,60 & 3,48 & 4,11 & 4,99 & 2,30 \\
\hline Fosforo disponible & $\mathrm{mg} / \mathrm{Kg}$ & 3,48 & 2,11 & 0,99 & 2,81 & 5,30 & 6,81 \\
\hline
\end{tabular}

\section{Conclusión}

En la Estación 1 se observó la presencia de suelos rojos, suelos con alto contenido de hierro, lo que corresponde a un plintosol, su textura es completamente arcillosa, hay lixiviación de hierro en las arcillas y presenta baja cantidad de materia orgánica. Esto tiene relación con lo planteado por el IGAC (1997), el cual realizó un estudio general de suelos del departamento del Cesar, dentro de este se destacaron los suelos de Valle, principalmente los de la asociación Fluventic Ustropents. Tipis Ustifluvents, estos son desarrollados de sedimentos aluviales (limos, arcillas y arenas).

En la estación 2 se evidenció la presencia de bioturbación debido a la presencia de lombrices, esto muestra que la salud del suelo es buena.
Hay pedregosidad superficial, por lo tanto, no se puede implementar arado, lo cual muestra que estos corresponden a un entisol; esto concuerda con lo planteado por Rangel, Carvajal-Cogollo y Arellano, quienes realizaron un estudio en el cual caracterizaron los suelos de la Serranía de Perijá y destacaron que en la región tropical predominan entisoles e inceptisoles.

Los suelos son ligeramente ácidos, lo que significa que son suelos productivos, es decir, suelos fértiles, esto es muy similar a lo planteado por Rangel, Carvajal-Cogollo y Arellano, quienes describieron el $\mathrm{pH}$ de estos suelos como neutro y ligeramente alcalino. En el uso del suelo se puede evidenciar un conflicto, ya que no se están aprovechando las bondades del este, sino que se utiliza principalmente para la ganadería ex- 
tensiva, tal como lo evidenció el IGAC (1997), quien también destacó que hay algunos cultivos de arroz y palma africana.

La textura predominante en estas dos estaciones fue la arcillosa y, en algunos casos, franco-arena-arcillosa, lo cual difiere un poco a lo descrito por Rangel, Carvajal-Cogollo y Arellano, quienes destacaron que en la región tropical predominan entisoles e inceptisoles, que tienen texturas franca y franca-arenosa.

Se puede concluir que los suelos de zona centro del departamento del Cesar son suelos muy productivos, los cuales se pueden utilizar para desarrollar diferentes actividades agrícolas que permitan que los habitantes de la región puedan encontrar una alternativa de generación de ingresos diferente a la actividad minera.

\section{Referencias}

Alcaldía La Jagua de Ibirico (2012). Plan de Salud Territorial del Municipio de La Jagua de Ibirico 2012-2015/2021.

Alcaldía Municipal de Becerril (2016). Plan de Manejo Ambiental (PMA). Proyecto: construcción del sistema de alcantarillado sanitario en los barrios Idema, El Carmen y San Martín del municipio de Becerril, Cesar.
Andrades, Moliner y Masaguer (2015). Prácticas de edafología. Métodos didácticos para análisis de suelos. Universidad de la Rioja.

Hernández, M. (2009). Memoria explicativa. Geología, plancha 48. Jagua de Ibirico. Escala 1:100.000, Ingeominas. Bogotá. A través de Universidad Nacional y ANH. Reconstrucción de la historia termal en los sectores de Luruaco y Cerro Cansona-cuenca del Sinú-San Jacinto y en el piedemonte occidental de la Serranía del Perijá entre Codazzi y la Jagua de Ibirico-cuenca de Cesar-Ranchería. Informe final. Cuenca Cesar-Ranchería.

Icontec (2004). Norma Técnica Colombiana NTC 5167. Productos para la industria agrícola. Productos orgánicos usados como abonos o fertilizantes y enmiendas de suelo. Bogotá, D. C.

IGAG (1997). Estudio general de suelos. Departamento del Cesar. Bogotá, D.C.

Jofpe, J. S. (1936). "Pedology". Rutgers University Press. New Brunswick, N.

J. A través de Jenny (1994). Factors of soil formation. A system of quantitative pedology. Dover Publications, INC. New York.

Rangel, Carvajal-Cogollo y Arellano. Suelos de la Serranía de Perijá.

Red Ormet, PNUD. Perfil productivo municipio. La Jagua de Ibirico.

Secretaría Departamental de Salud del Cesar (2010). Análisis de situación de salud de fronteras (ASIS). Municipios fronterizos con Venezuela. Departamento del Cesar. Eje 1, La Guajira, Cesar y Zulia (Colombia-Venezuela). 